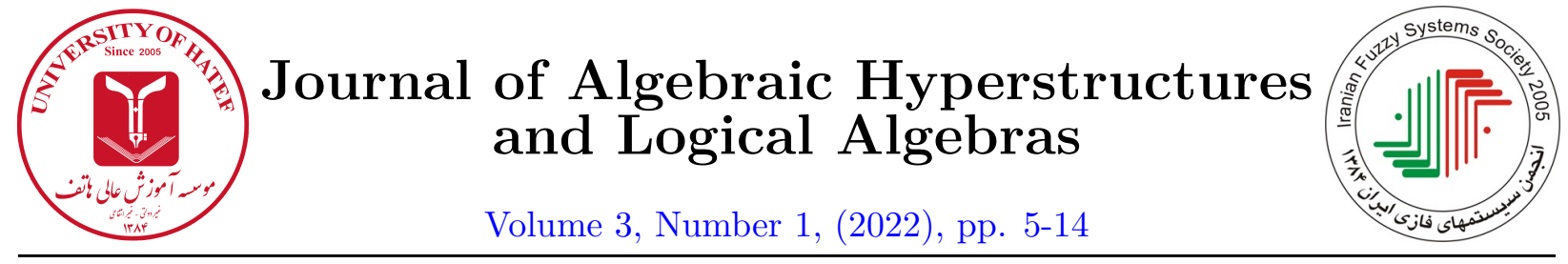

\title{
A lattice-theoretical approach to extensions of filters in algebras of substructural logic
}

\author{
D. Šalounová ${ }^{1}$ and J. Rachůnek ${ }^{2}$ \\ ${ }^{1}$ Department of Mathematical Methods in Economy, Faculty of Economics, VŠB-Technical University \\ Ostrava, Sokolská 33, 70121 Ostrava, Czech Republic \\ ${ }^{2}$ Department of Algebra and Geometry, Faculty of Sciences, Palacký University, 17. listopadu 12, 77146 \\ Olomouc, Czech Republic
}

dana.salounova@vsb.cz, jiri.rachunek@upol.cz

"This paper is dedicated to Professor Antonio Di Nola on the occasion of his 75th birthday."

\begin{abstract}
Commutative bounded integral residuated lattices (residutaed lattices, in short) form a large class of algebras containing algebras which are algebraic counterparts of certain propositional fuzzy logics. The paper deals with the so-called extended filters of filters of residuated lattices. It is used the fact that the extended filters of filters associated with subsets coincide with those associated ones with corresponding filters. This makes it possible to investigate the set of all extended filters of residuated lattices within the Heyting algebras of their filters by means of the structural methods of the theory of such algebras.
\end{abstract}

\section{Article Information}

Corresponding Author:

D. Šalounová;

Received: August 2021;

Accepted: Invited paper;

Paper type: Original.

\section{Keywords:}

Residuated lattice, Heyting algebra, relative Stone algebra.

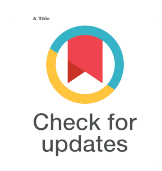

\section{Introduction}

Extended filters associated with subsets of bounded commutative $R \ell$ - monoids (= bounded integral commutative residuated lattices satisfying divisibility) have been introduced and investigated in [8]. It was shown there that if $M$ is an $R \ell$-monoid and $B$ is a subset of $M$, then the extended filter of $M$ associated with $B$ coincides with the extended filter of $M$ associated with the filter $\langle B\rangle$ of $M$ generated by $B$. This gives us the opportunity to study, without loss of generality, extended filters of $R \ell$-monoids only within the (complete) lattices of their filters. It is known that the lattices of filters and congruences of bounded $R \ell$-monoids are mutually isomorphic, e.g.

https://doi.org/10.52547/HATEF.JAHLA.3.1.2 
[9]. Moreover, the congruence lattices of bounded $R \ell$-monoids are Heyting algebras because the signature of $R \ell$-monoids contains a lattice reduct. Hence the lattices of filters of $R \ell$-monoids are also Heyting algebras. Moreover, if $M$ is an $R \ell$-monoid, then in the lattice $\mathcal{F}(M)$ of filters of $M$, extended filters associated with filters coincide with relative pseudocomplements in the lattice $\mathcal{F}(M)$.

Therefore it is not necessary to prove some assertions concerning extended filters directly using methods of the theory of $R \ell$-monoids, e.g. [8], but it suffices to show that they are special properties of Heyting algebras.

Similarly it is possible to apply properties of Heyting algebras to the lattices of filters of more general residuated lattices.

Commutative bounded integral residuated lattices (residuated lattices, in short) form a large class of algebras which contains e.g. algebras that are algebraic counterparts of some propositional many-valued and fuzzy logics. We can consider as particular cases of residuated lattices: $M T L-$ algebras [5] that are algebras of the monoidal $t$-norm based logic, $B L$-algebras [7], i.e. algebras of Hájek's basic fuzzy logic, $M V$-algebras [2] that are an algebraic semantics of the Eukasiewicz infinite valued logic. Moreover, Heyting algebras, i.e. algebras of the intuitionistic logic can be also recognized as residuated lattices. Therefore residuated lattices can be considered as algebras of the substructural logic FL, the more general logic that contains the mentioned non-classical logics as particular cases [6]. The deductive systems of those logics correspond to filters of their algebraic counterparts.

The main goal of the paper is to show that many of results concerning filters of all residuated lattices can be also obtained as simple applications of appropriate properties of Heyting algebras.

In Section 2 we recall the notion of a commutative bounded integral residuated lattice (a residuated lattice in short) as a common generalization of algebras of certain fuzzy logics.

In Section 3 we deal with extended filters of filters associated with subsets of residuated lattices. We use the fact that if $B$ is a subset of a residuated lattice $M$ and $F$ is a filter of $M$, then the extended filter $E_{F}(B)$ associated with $B$ coincides with the filter $E_{F}(\langle B\rangle)$ associated with the filter $\langle B\rangle$ of $M$ generated by $B$. This enables us to investigate, without loos of generality, only extended filters associated with filters within the Heyting lattice $\mathcal{F}(M)$ of filters of $M$ using the technique of Heyting algebras. The properties of extended filters are then particular cases of properties of Heyting algebras.

In Section 4 we investigate the sets of extended filters of type $E_{F}(K)$ where $F$ and $K$ are filters of $M$ and $F$ or $K$ are fixed. The results are again obtained as particular cases of those of Heyting algebras.

\section{Preliminaries}

A commutative bounded integral residuated lattice is an algebra $M=(M ; \odot, \vee, \wedge, \rightarrow, 0,1)$ of type $\langle 2,2,2,2,0,0\rangle$ satisfying the following conditions.

(i) $(M ; \odot, 1)$ is a commutative monoid.

(ii) $(M ; \vee, \wedge, 0,1)$ is a bounded lattice.

(iii) $x \odot y \leq z$ if and only if $x \leq y \rightarrow z$, for any $x, y, z \in M$.

In what follows, by a residuated lattice we will mean a commutative bounded integral residuated lattice. 
For any residuated lattice $M$ we define the unary operation (negation) "-" on $M$ by $x^{-}:=x \rightarrow 0$ for any $x \in M$.

Recall that algebras of logics mentioned in Introduction are characterized in the class of residuated lattices as follows:

A residuated lattice $M$ is

(a) an $M T L$-algebra [5] if $M$ satisfies the identity of pre-linearity

(iv) $(x \rightarrow y) \vee(y \rightarrow x)=1$;

(b) involutive if $M$ satisfies the identity of double negation

(v) $x^{--}=x$;

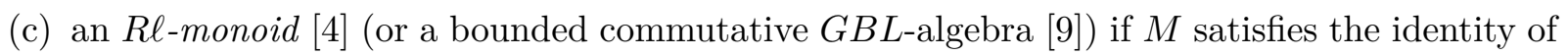
divisibility

(vi) $(x \rightarrow y) \odot x=x \wedge y$;

(d) a BL-algebra [7] if $M$ satisfies both (a) and (c);

(e) an $M V$-algebra [2] if $M$ is an involutive $B L$-algebra;

(f) a Heyting algebra [1] if the operations " $\odot$ " and " $\wedge$ " coincide on $M$.

Lemma 2.1. [3, 9] Let $M$ be a residuated lattice. Then for any $x, y, z \in M$ we have:

(i) $x \leq y \Longrightarrow y^{-} \leq x^{-}$,

(ii) $x \odot y \leq x \wedge y$,

(iii) $\quad(x \rightarrow y) \odot x \leq y$,

(iv) $\quad x \leq x^{--}$,

(v) $x^{---}=x^{-}$,

(vi) $\quad x \leq y \Longrightarrow y \rightarrow z \leq x \rightarrow z$,

(vii) $x \leq y \Longrightarrow z \rightarrow x \leq z \rightarrow y$,

(viii) $\quad x \odot(y \vee z)=(x \odot y) \vee(x \odot z)$,

$(i x) \quad x \vee(y \odot z) \geq(x \vee y) \odot(x \vee z)$,

$(x) \quad x \rightarrow(y \rightarrow z)=(x \odot y) \rightarrow z$.

Let $M$ be a residuated lattice and $\emptyset \neq F \subseteq M$, then $F$ is called a filter of $M$ if for any $x, y \in F$ and $z \in M$ :

1. $x \odot y \in F$;

2. $x \leq z$ imply $z \in F$.

Let $D \subseteq M$, then $D$ is called a deductive system of $M$ if 
3. $1 \in D$;

4. $x \in D, x \rightarrow y \in D$ imply $y \in D$.

Proposition 2.2. Let $M$ is a residuated lattice and $\emptyset \neq H \subseteq M$, then $H$ is a filter of $M$ if and only if $H$ is a deductive system.

Proof. Let $H$ be a filter of $M$. obviously $1 \in H$. Let $x \in H, y \in M$ and $x \rightarrow y \in H$. Then $(x \rightarrow y) \odot x \in H$. Since $(x \rightarrow y) \odot x \leq y$, we have $y \in H$.

Conversely, let $H$ be a deductive system of $M$ and $x, y \in H$. Then, by Lemma 2.1(x), $x \rightarrow(y \rightarrow(x \odot y))=(x \odot y) \rightarrow(x \odot y)=1 \in H$ thus $y \rightarrow(x \odot y) \in H$, therefore also $x \odot y \in H$. If $x \in H, z \in M$ and $x \leq z$, then $x \rightarrow z=1 \in H$, hence $z \in H$.

Denote by $\mathcal{F}(M)$ the set of all filters of a residuated lattice $M$. Then $\mathcal{F}(M)$ ordered by set inclusion is a complete lattice in which infima are equal to the set intersections.

If $B \subseteq M$, denote by $\langle B\rangle$ the filter of $M$ generated by $B$. Then for $\emptyset \neq B \subseteq M$ we have

$$
\langle B\rangle=\left\{z \in M: z \geq b_{1} \odot \cdots \odot b_{n}, \text { where } n \in \mathbb{N}, b_{1}, \ldots, b_{n} \in B\right\} .
$$

\section{Extended filters}

Extended filters of filters of $R \ell$-monoids, i.e. of residuated lattices satisfying divisibility, have been defined and studied in [8], using the direct methods of $R \ell$-monoids. Here we generalize extended filters of filters to arbitrary (commutative bounded integral) residuated lattices and show that results concerning extended filters can be simply obtained as special cases of properties of Heyting algebras.

In accordance with [8] we use the following denotation: If $M$ is a residuated lattice, $F \in \mathcal{F}(M)$ and $B \subseteq M$, put

$$
E_{F}(B):=\{x \in M: x \vee b \in F \text { for every } b \in B\} .
$$

Theorem 3.1. Let $M$ be a residuated lattice, $F \in \mathcal{F}(M)$ and $B \subseteq M$. Then $E_{F}(B) \in \mathcal{F}(M)$ and $F \subseteq E_{F}(B)$.

Proof. For any $b \in B, 1 \vee b=1 \in F$, thus $1 \in E_{F}(B)$.

Let $x, x \rightarrow y \in E_{F}(B)$. Then $(b \vee x) \odot(b \vee(x \rightarrow y)) \leq b \vee(x \odot(x \rightarrow y)) \leq b \vee y$, hence $b \vee y \in F$ for any $b \in B$, thus $y \in E_{F}(B)$. Therefore $E_{F}(B) \in \mathcal{F}(M)$.

Let $x \in F$. Then $x \vee b \in F$ for every $b \in B$, hence $x \in E_{F}(B)$.

$E_{F}(B)$ will be called the extended filter of a filter $F$ associated with a subset $B$.

Theorem 3.2. If $M$ is a residuated lattice, $B \subseteq M$ and $\langle B\rangle$ is the filter of $M$ generated by $B$, then $E_{F}(B)=E_{F}(\langle B\rangle)$ for any $F \in \mathcal{F}(M)$.

Proof. Let us show firstly that if $F \in \mathcal{F}(M)$ and $B \subseteq C \subseteq M$, then $E_{F}(C) \subseteq E_{F}(B)$. Let $x \in E_{F}(C)$. Then $x \vee c \in F$ for every $c \in C$, thus $x \vee b \in F$ for every $b \in B$, that means $x \in E_{F}(B)$. Therefore $E_{F}(\langle B\rangle) \subseteq E_{F}(B)$ for any $B \subseteq M$.

Let now $x \in E_{F}(B)$, i.e. $x \vee b \in F$ for any $b \in B$. If $z \in\langle B\rangle$ then there are $b_{1}, \ldots, b_{n} \in B$ such that $z \geq b_{1} \odot \cdots \odot b_{n}$. Hence $x \vee z \geq x \vee\left(b_{1} \odot \cdots \odot b_{n}\right) \geq\left(x \vee b_{1}\right) \odot \cdots \odot\left(x \vee b_{n}\right)$. Since $x \vee b_{i} \in F, i=1, \ldots, n$, and $F \in \mathcal{F}(M)$, we get $x \vee z \in F$. That means $x \in E_{F}(\langle B\rangle)$. 
Recall the notions of pseudocomplemented lattices and Heyting algebras [1]. Let $L$ be a lattice with 0 . An element $a \in L$ is pseudocomplemented if there is a largest element in $L$ which is disjoint with $a$. If such an element exists, it is denoted by $a^{*}$ and is called the pseudocomplement of $a$. That means $a \wedge x=0$ iff $x \leq a^{*}$, for each $x \in L$. A pseudocomplemented lattice is a lattice with 0 in which every element has a pseudocomplement. Let $L$ be a lattice and $a, b \in L$. If there is a largest $x \in L$ such that $a \wedge x \leq b$, then this element is denoted by $a \rightarrow b$ and is called the relative pseudocomplement of a with respect to $b$. A Heyting algebra is a lattice with 0 in which $a \rightarrow b$ exists for each $a, b \in L$. Heyting algebras satisfy the infinite distributive law: If $L$ is a Heyting algebra, $\left\{b_{i}: i \in I\right\} \subseteq L$ and $\bigvee_{i \in I} b_{i}$ exists then for each $a \in L, \bigvee_{i \in I}\left(a \wedge b_{i}\right)$ exists and $a \wedge \bigvee_{i \in I} b_{i}=\bigvee_{i \in I}\left(a \wedge b_{i}\right)$

A Heyting algebra is called a relative Stone algebra if it satisfies the identity $(x \rightarrow y) \vee(y \rightarrow$ $x)=1$. We will say that a Heyting algebra is representable if it is a subdirect product of linearly ordered Heyting algebra. By [10], every relative Stone algebra is representable.

Now we go back to extended filters of residuated lattices. Based on Theorem 3.2, in the sequel we will investigate, without loss of generality, $E_{F}(B)$ only for $B \in \mathcal{F}(M)$.

The lattice $\mathcal{F}(M)$ is isomorphic to the congruence lattice on $M$ which is, as the congruence lattice on an algebra with underlying lattice reduct, a Heyting algebra, too. Moreover, it is easy to check, that $E_{F}(K)=K \rightarrow F$, for every $F, K \in \mathcal{F}(M)$. Firstly, we show that $K \cap E_{F}(K) \subseteq F$. If $x \in K \cap E_{F}(K)$ than $x \vee x=x \in F$. Further, let us assume $K \cap X \subseteq F$ for some $X \in \mathcal{F}(M)$. Then $K \cap X \subseteq K \cap F \subseteq K \cap E_{F}(K)$, by Theorem 3.1. It implies $X \subseteq E_{F}(K)$.

The following theorem contains a list of properties of extended filters of residuated lattices which are direct consequences of corresponding ones for elements of Heyting algebras (see e.g. [1, Theorem IX.3]).

Theorem 3.3. Let $M$ be a residuated lattice and $F, K, G, L, F_{i}, K_{i} \in \mathcal{F}(M), i \in I$. Then:

$$
\begin{array}{ll}
\text { 1. } & K \cap E_{F}(K) \subseteq F \\
\text { 2. } & K \subseteq E_{F}\left(E_{F}(K)\right) ; \\
\text { 3. } & F \subseteq E_{F}(K) ; \\
\text { 4. } & F \subseteq G \Longrightarrow E_{F}(K) \subseteq E_{G}(K) ; \\
\text { 5. } & F \subseteq G \Longrightarrow E_{K}(G) \subseteq E_{K}(F) ; \\
\text { 6. } & K \cap E_{F}(K)=K \cap F ; \\
\text { 7. } & E_{F}(K)=M \Longleftrightarrow K \subseteq F ; \\
\text { 8. } & E_{F}\left(E_{F}(G)\right) \cap E_{F}(G)=F ; \\
\text { 9. } & F \subseteq G \Longrightarrow E_{F}(G) \cap G=F ; \\
\text { 10. } & E_{F}\left(E_{F}\left(E_{F}(K)\right)\right)=E_{F}(K) ; \\
\text { 11. } & K \subseteq L, E_{F}(K)=F \Longrightarrow E_{F}(L)=F ; \\
\text { 12. } & E_{E_{M}(L)}(K)=E_{M}(K \cap L) ; \\
\text { 13. } & E_{E_{F}(K)}(L)=E_{E_{F}(L)}(K) ;
\end{array}
$$


14. $E_{F}(K)=F \Longrightarrow E_{F}\left(E_{F}(K)\right)=M$;

15. $\bigcap_{i \in I} E_{F_{i}}(K)=E_{\bigcap\left\{F_{i}: i \in I\right\}}(K)$;

16. $E_{F}\left(\bigvee_{i \in I} K_{i}\right)=\bigcap_{i \in I} E_{F}\left(K_{i}\right)$.

Proof. As examples, we can verify, e.g., properties 8 and 15. For completeness we give also the proofs of the corresponding assertions for Heyting algebras.

8. Let $A$ be a Heyting algebra, $x, y \in A$. Then $(x \rightarrow y) \rightarrow y \geq y, x \rightarrow y \geq y$, hence $((x \rightarrow y) \rightarrow y) \wedge(x \rightarrow y) \geq y$. At the same time, by the definition of a Heyting algebra, $(x \rightarrow y) \wedge((x \rightarrow y) \rightarrow y) \leq y$. Thus $((x \rightarrow y) \rightarrow y) \wedge(x \rightarrow y)=y$.

Therefore, $E_{F}\left(E_{F}(G)\right) \cap E_{F}(G)=\left(E_{F}(G) \rightarrow F\right) \cap E_{F}(G)=((G \rightarrow F) \rightarrow F) \cap(G \rightarrow F)=F$.

15. For Heyting algebras, which need not be complete, we prove a little more general assertion: If $A$ is a Heyting algebra, $x, y_{i} \in A, i \in I$, and $\bigwedge_{i \in I} y_{i}$ exists, then $\bigwedge_{i \in I}\left(x \rightarrow y_{i}\right)$ also exists and $x \rightarrow \bigwedge_{i \in I} y_{i}=\bigwedge_{i \in I}\left(x \rightarrow y_{i}\right)$

We have $x \rightarrow \bigwedge_{i \in I} y_{i} \leq x \rightarrow y_{j}$ for each $j \in I$, and if $z \in A$ is such that $z \leq x \rightarrow y_{j}$, for each $j \in I$, then $x \wedge z \leq y_{j}$, for each $j \in I$, hence $z \leq x \rightarrow \bigwedge_{i \in I} y_{i}$, and so $x \rightarrow \bigwedge_{i \in I} y_{i}=\bigwedge_{i \in I}\left(x \rightarrow y_{i}\right)$.

From this it follows immediately: $\bigcap_{i \in I} E_{F_{i}}(K)=\bigcap_{i \in I}\left(K \rightarrow F_{i}\right)=K \rightarrow \bigcap_{i \in I} F_{i}=E_{\bigcap\left\{F_{i}: i \in I\right\}}(K)$.

\section{Sets of extended filters with a fixed filter}

Now we will deal with the sets $E_{F}(K)$ where $F$ and $K$, respectively, are fixed.

Let $A$ be a Heyting algebra and $a \in A$. Put

$$
E(a):=\{a \rightarrow x: x \in A\} .
$$

In particular, if $M$ is a residuated lattice and $K \in \mathcal{F}(M)$, then $E(K)=\{K \rightarrow F: F \in \mathcal{F}(M)\}=$ $\left\{E_{F}(K): F \in \mathcal{F}(M)\right\}$.

Recall that if $A$ is a Heyting algebra, $a, x_{i} \in A, i \in I$ and $\bigwedge_{i \in I} x_{i}$ exists, then $\bigwedge_{i \in I}\left(a \rightarrow x_{i}\right)$ also exists and

$$
\bigwedge_{i \in I}\left(a \rightarrow x_{i}\right)=a \rightarrow \bigwedge_{i \in I} x_{i}
$$

Therefore we obtain the following assertion.

Proposition 4.1. a) If $A$ is a Heyting algebra, $a \in A$ and $x_{i} \in E(a), i \in I$, then $\bigwedge_{i \in I} x_{i}$ exists if and only if $\bigwedge_{i \in I} x_{i}$ exists, and in such a case $\bigwedge_{i \in I} x_{E(a)} x_{i}=\bigwedge_{i \in I} x_{i}$.

b) If $A$ is a complete Heyting algebra, then for any $a \in A, E(a)$ is a complete lattice which is a complete inf-subsemilattice of $A$. 
Theorem 4.2. If $M$ is a residuated lattice and $K \in \mathcal{F}(M)$, then $(E(K), \subseteq)$ is a complete lattice which is a complete inf-subsemilattice of the lattice $\mathcal{F}(M)$.

Now we will describe a large class of residuated lattices $M$ such that, for every $K \in \mathcal{F}(M), E(K)$ is not only an inf-sublattice but also a sublattice of $\mathcal{F}(M)$.

Proposition 4.3. Every linearly ordered Heyting algebra satisfies the identity

$$
x \rightarrow(y \vee z)=(x \rightarrow y) \vee(x \rightarrow z) .
$$

Proof. Let $A$ be a linearly ordered Heyting algebra and $x, y, z \in A$.

a) If $x \leq y \leq z$, then $x \rightarrow(y \vee z)=x \rightarrow z=1$ and $(x \rightarrow y) \vee(x \rightarrow z)=1 \vee 1=1$.

b) If $y \leq z \leq x$. Then $x \rightarrow(y \vee z)=x \rightarrow z$ and $x \rightarrow y \leq x \rightarrow z$. So $(x \rightarrow y) \vee(x \rightarrow z)=x \rightarrow z$.

c) If $z \leq x \leq y$, then $x \rightarrow(y \vee z)=x \rightarrow y=1$, and $(x \rightarrow y) \vee(x \rightarrow z)=1 \vee(x \rightarrow z)=1$.

Corollary 4.4. If $A$ is a representable Heyting algebra or a relative Stone algebra then $A$ satisfies (1).

Corollary 4.5. If $A$ is a representable Heyting algebra or a relative Stone algebra, and $a \in A$, then $E(a)$ is a sublattice of $A$.

Theorem 4.6. If $M$ is a residuated lattice such that $\mathcal{F}(M)$ is a representable Heyting algebra or a relative Stone algebra and $K \in \mathcal{F}(M)$, then $E(K)$ is a sublattice of the lattice $\mathcal{F}(M)$.

Let now $A$ be a Heyting algebra and $a \in A$. Put $E_{a}:=\{x \rightarrow a: x \in A\}$. In particular, if $M$ is a residuated lattice and $K \in \mathcal{F}(M)$, then $E_{K}=\{F \rightarrow K: F \in \mathcal{F}(M)\}=\left\{E_{F}(K): K \in \mathcal{F}(M)\right\}$.

Recall that if $A$ is a Heyting algebra, $a \in A$ and $x_{i} \in E(a), i \in I$, then $\bigwedge_{i \in I} x_{E}$ exists if and only if $\bigwedge_{i \in I} x_{i}$ exists, and in such a case $\bigwedge_{i \in I} x_{E(a)} x_{i}=\bigwedge_{i \in I} x_{i}$.

Now consider the mapping $\varphi: A \longrightarrow A$ such that $\varphi(x)=(x \rightarrow a) \rightarrow a$. Then for every $x, y \in A$ :

1. $(x \rightarrow a) \rightarrow a \geq x$, thus $x \leq \varphi(x)$;

2. If $x \leq y$, then $x \rightarrow a \geq y \rightarrow a$, hence $(x \rightarrow a) \rightarrow a \leq(y \rightarrow a) \rightarrow a$, and so $\varphi(x) \leq \varphi(y)$;

3. $\varphi(\varphi(x))=\varphi((x \rightarrow a) \rightarrow a)=(((x \rightarrow a) \rightarrow a) \rightarrow a) \rightarrow a=(x \rightarrow a) \rightarrow a=\varphi(x)$.

Hence $\varphi$ is a closure operator on $A$ and $E_{a}$ is the set of closed elements with respect to $\varphi$.

From this we get the assertion concerning infima of arbitrary systems of elements in $E_{a}$.

The following assertion is now an immediate consequence.

Proposition 4.7. If $A$ is a complete Heyting algebra and $a \in A$, then $E_{a}$ is a complete lattice which is a complete inf-subsemilattice of $A$.

Theorem 4.8. If $M$ is a residuated lattice and $K \in \mathcal{F}(M)$, then $E_{K}$ is a complete inf-subsemilattice of the lattice $\mathcal{F}(M)$.

Now we will show further properties of the sets $E_{a}$. If $A$ is a pseudocomplemented distributive lattice, denote by $\operatorname{Reg}(A):=\left\{x \in A: x^{* *}=x\right\}$, the set of regular elements in $A$. By Glivenko's theorem, see e.g. [1], $\operatorname{Reg}(A)$ is a Boolean algebra with respect to the induced order. 
Proposition 4.9. If $A$ is a Heyting algebra and $a \in A$, then $E_{a}$ is a Boolean algebra with respect to the induced order.

Proof. By [1, Theorem IX.28], the interval $[a, 1]$ is a Heyting algebra, hence also a pseudocomplemented distributive lattice. If $x \rightarrow a$, where $x \in A$, is an arbitrary element in $E_{a}$, then its pseudocomplement in $[a, 1]$ is $(x \rightarrow a)^{*}=(x \rightarrow a) \rightarrow a$. Thus in $[a, 1]$ we obtain $(x \rightarrow a)^{* *}=((x \rightarrow a) \rightarrow a) \rightarrow a=x \rightarrow a$, and so $x \rightarrow a \in \operatorname{Reg}([a, 1])$.

Therefore by Glivenko's theorem we get that $E_{a}$ is a Boolean algebra with respect to the induced order.

The following theorem is now an immediate consequence.

Theorem 4.10. If $M$ is a residuated lattice and $K \in \mathcal{F}(M)$, then $E_{K}$ ordered by inclusion is a Boolean algebra.

Proposition 4.11. Every linearly ordered Heyting algebra satisfies the identity

$$
(y \wedge z) \rightarrow x=(y \rightarrow x) \vee(z \rightarrow x) .
$$

Proof. Let $A$ be a linearly ordered Heyting algebra and $x, y, z \in A$.

a) If $x \leq y \leq z$. Then $(y \wedge z) \rightarrow x=y \rightarrow x$ and $z \rightarrow x \leq y \rightarrow x$. So $(y \rightarrow x) \vee(z \rightarrow x)=y \rightarrow x$.

b) If $y \leq z \leq x$, then $(y \wedge z) \rightarrow x=y \rightarrow x=1$ and $(y \rightarrow x) \vee(z \rightarrow x)=1 \vee 1=1$.

c) If $z \leq x \leq y$. Then $(y \wedge z) \rightarrow x=z \rightarrow x=1$ and $(y \rightarrow x) \vee(z \rightarrow x)=(y \rightarrow x) \vee 1=1$.

Corollary 4.12. Every representable Heyting algebra satisfies (2).

Corollary 4.13. Every relative Stone algebra satisfies (2).

Corollary 4.14. If $A$ is a relative Stone algebra, or a representable Heyting algebra, and $a \in A$, then $E_{a}$ is a sublattice of the lattice $A$.

Theorem 4.15. If $M$ is a residuated lattice such that $\mathcal{F}(M)$ is a relative Stone algebra or a representable Heyting algebra and $K \in \mathcal{F}(M)$, then $E_{K}$ is a sublattice of the lattice $\mathcal{F}(M)$.

From the other side, now we will show some classes of residuated lattice $M$ that contain filters $K$ such that $E(K)$ and $E_{K}$, respectively, are not sublattices of $\mathcal{F}(M)$.

Proposition 4.16. Let $A$ be a Heyting algebra such that the subset $A \backslash\{1\}$ has a greatest element $a$ and let there exist $b, c \in A$ such that $b<a, c<a$ and $b \vee c=a$. Then $E(a)$ is not a sublattice of $A$.

Proof. Let $A$ contain such elements $a, b$ and $c$. Then $a \rightarrow y=y$ for any $y<a$ and $a \rightarrow a=1=$ $a \rightarrow 1$, hence $a \notin E(a)$, but $b, c \in E(a)$. Therefore in the lattice $E(a)$ we have $b \vee_{E(a)} c=1$, that means $E(a)$ is not a sublattice of $A$.

Example 4.17. Let $M$ be the lattice in the following figure. Then $M$ is a Heyting algebra with the relative pseudocomplements in the following table. 


\begin{tabular}{|c|ccccc|}
\hline$\rightarrow$ & 0 & $a$ & $b$ & $c$ & 1 \\
\hline 0 & 1 & 1 & 1 & 1 & 1 \\
$a$ & 0 & 1 & 1 & 1 & 1 \\
$b$ & 0 & $c$ & 1 & $c$ & 1 \\
$c$ & 0 & $b$ & $b$ & 1 & 1 \\
1 & 0 & $a$ & $b$ & $c$ & 1 \\
\hline
\end{tabular}

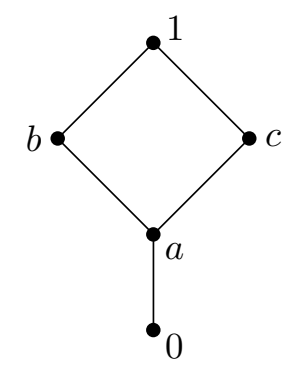

If we put $\odot=\wedge$, then $M=(M ; \vee, \wedge, \odot, \rightarrow, 0,1)$ is a residuated lattice. Since the filters of the residuated lattice $M$ are precisely the lattice filters of $M$, we get $\mathcal{F}(M)=\left\{F_{0}, F_{a}, F_{b}, F_{c}, F_{1}\right\}$, where $F_{0}=M=\{0, a, b, c, 1\}, F_{a}=\{a, b, c, 1\}, F_{b}=\{b, 1\}, F_{c}=\{c, 1\}, F_{1}=\{1\}$. Hence the lattice $\mathcal{F}(M)$ is anti-isomorphic to the lattice $M$ on the following figure:

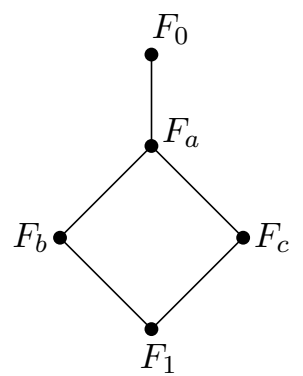

Then we get $E\left(F_{a}\right)=\left\{F_{1}, F_{b}, F_{c}, F_{0}\right\}$ and according to Proposition 4.16, $E\left(F_{a}\right)$ is not a sublattice of $\mathcal{F}(M)$.

Proposition 4.18. Let $A$ be a Heyting algebra which contains elements $a, b, c, d$ such that $a<$ $b<d<1, a<c<d<1, b \wedge c=a, b \vee c=d, d$ is the greatest element in $A \backslash\{1\}$ and $a$ is the greatest element in $A \backslash\{b, c, d, 1\}$. Then $E_{a}$ is not a sublattice of the lattice $A$.

Proof. Let $A$ contain such elements $a, b, c$ and $d$. Let $z \rightarrow a=d$ for some $z \in A$. Then $z \rightarrow a \neq 1$, hence $z \not \leq a$, and thus $z \in\{b, c, d, 1\}$. At the same time $b \rightarrow a=c, c \rightarrow a=b, d \rightarrow a=a, 1 \rightarrow$ $a=a$, a contradiction. Therefore $d \notin E_{a}$, while $b, c \in E_{a}$. From this we get $b \vee_{E_{a}} c \neq b \vee_{A} c$, and so $E_{a}$ is not a sublattice of the lattice $A$.

Example 4.19. Let $M$ be again the residuated lattice from Example 4.17. Then $E_{F_{1}}=\left\{F_{1}, F_{b}, F_{c}, F_{0}\right\}$, and hence $E_{F_{1}}$ is not a sublattice of the lattice $\mathcal{F}(M)$.

\section{References}

[1] R. Balbes, P. Dwinger, Distributive lattices, University of Missouri Press, Columbia, Missouri, 1974.

[2] R.L.O. Cignoli, I.M.L. D'Ottaviano, D. Mundici, Algebraic foundation of many-valued reasoning, Kluwer Academic Publishers, Dordrecht - Boston - London, 2000.

[3] L.C. Ciungu, Classes of residuated lattices, Annals of the University of Craiova, Mathematics and Computer Science Series, 33 (2006), 189-207. 
[4] A. Dvurečenskij, J. Rachůnek, Probabilistic averaging in bounded commutative residuated $\ell$ monoids, Discrete Mathematics, 306 (2006), 1317-1326.

[5] F. Esteva, L. Godo, Monoidal t-norm based logic: Towards a logic for left-continuous t-norms, Fuzzy Sets and Systems, 124 (2001), 271-288.

[6] N. Galatos, P. Jipsen, T. Kowalski, H. Ono, Residuated lattices: An algebraic glimpse at substructural logics, Elsevier Studies in Logic and Foundations. Elsevier, Amsterdam, 2007.

[7] P. Hájek, Metamathematics of fuzzy logic, Kluwer, Amsterdam, 1998.

[8] M. Haveshki, M. Mohamadhasani, Extended filters in bounded commutative R $\ell$-monoids, Soft Computing, 16 (2012), 2165-2173.

[9] P. Jipsen, C. Tsinakis, A survey of residuated lattices, In: Ordered Algebraic Structures (ed. J. Martinez), Kluwer Acad. Publ. Dordrecht, 2002, 19-56.

[10] T. Katriňák, Remarks on the W.C. Nemitz's paper "Semi-Boolean lattices", Notre Dame Journal of Formal Logic, 11 (1970), 425-430. 